\title{
Effect of turbulence on the wake of a wall-mounted cube
}

\author{
R. Jason Hearst, Guillaume Gomit and Bharathram \\ Ganapathisubramani $\dagger$ \\ Engineering and the Environment, University of Southampton, Southampton, SO17 1BJ, \\ United Kingdom
}

(Received $\mathrm{xx}$; revised $\mathrm{xx}$; accepted $\mathrm{xx}$ )

The influence of turbulence on the flow around a wall-mounted cube immersed in a turbulent boundary layer is investigated experimentally with particle image velocimetry and hot-wire anemometry. Free-stream turbulence is used to generate turbulent boundary layer profiles where the normalised shear at the cube height is fixed, but the turbulence intensity at the cube height is adjustable. The free-stream turbulence is generated with an active grid and the turbulent boundary layer is formed on an artificial floor in a wind tunnel. The boundary layer development Reynolds number $\left(R e_{x}\right)$ and the ratio of the cube height $(h)$ to the boundary layer thickness $(\delta)$ are held constant at $R e_{x}=1.8 \times 10^{6}$ and $h / \delta=0.47$. It is demonstrated that the stagnation point on the upstream side of the cube and the reattachment length in the wake of the cube are independent of the incoming profile for the conditions investigated here. In contrast, the wake length monotonically decreases for increasing turbulence intensity but fixed normalised shear - both quantities measured at the cube height. The wake shortening is a result of heightened turbulence levels promoting wake recovery from high local velocities and the reduction in strength of a dominant shedding frequency.

Key words: (to be entered by typesetter)

\section{Introduction}

The flow around a wall-mounted cube is a canonical problem of great interest. Practically, it both models a single roughness element and can be scaled to extract mean pressure and velocity fields around objects in the atmospheric boundary layer (Lim et al. 2007). Particular applications include flow around buildings, wind turbines, and other large scale construction projects. In such cases, it is often difficult to measure the freestream velocity $\left(U_{\infty}\right)$ but measurements at the building height are quite attainable. As such, any parameterisation of such a problem is preferably addressed with the local flow characteristics.

The seminal work by Castro \& Robins (1977) is perhaps one of the most comprehensive investigations of the flow around wall-mounted cubes to date. One aspect of this work focussed on the effect of turbulence on the cube wake by changing the ratio of the cube height $(h)$ to the boundary layer thickness $(\delta)$. Castro \& Robins (1977) found that for $h / \delta \lesssim 0.7$ the flow achieved a certain degree of Reynolds number independence in terms of the reattachment length behind the cube and the flow field characteristics; such cubes

$\dagger$ Email address for correspondence: g.bharath@southampton.ac.uk 
are smaller than $\delta$ and thus completely immersed within the turbulence of the boundary layer. This appeared to be related to the flow attaching to the top of the cube, which in turn was associated with the turbulence level at the cube height $\left(u_{h}^{\prime} / U_{h}\right)$; the subscript $\cdot h$ denotes a quantity measured at the cube height. Castro \& Robins (1977) changed $h / \delta$ by placing cubes of different height in the same turbulent boundary layer (TBL), which resulted in the top of the cubes intersecting different locations in the boundary layer profile. As such, they postulated the results must also be dependent on the shear at the cube height $\left(\tau_{h}=\partial U /\left.\partial y\right|_{h}\right)$. However, $u_{h}^{\prime} / U_{h}$ and $\tau_{h}$ are inherently coupled when $h$ is changed and the boundary layer profile is maintained, which led them to suggest "studies of, for example, the separate effects of turbulence and shear would be desirable" (Castro \& Robins 1977, p. 308).

The integral length scale $(L)$ of the incoming turbulence may also play a role in the flow around a cube. Saathoff \& Melbourne (1997) demonstrated that the pressure fluctuations inside the separation bubble that formed on the leading edge of a thick flat plate increased with $L / h$, while the effect of the turbulence intensity was to bring the attachment point of this bubble closer to the leading edge; $h$ is the thickness of the flat plate. However, this was for a factor of 5 change in $L / h$. For the range $0.36<L / h<1.95$, Hillier \& Cherry (1981) found that the integral scale did not significantly influence the separation bubble on a flat plate. Furthermore, the large eddy simulations (LES) of a wall-mounted square cylinder (aspect ratio 4:1) performed by Li et al. (2014) also identified that $L$ did not have a significant impact on the forces felt by the cylinder for $0.2<L_{h} / D<0.5$, where $D$ was the cylinder width. Thus, if the integral scale can be kept small relative to the characteristic dimension of the cube, then $L$ may not be a parameter of significant influence.

The dynamics of the flow around cubes and square cylinders has also been investigated in some detail. Baker $(1979,1980)$ investigated the horseshoe vortex that forms around these bodies for both laminar and turbulent incoming conditions using smoke visualisations and pressure taps. Ballio et al. (1998) developed these studies further and found that for turbulent flows the dimensions of the vortex system were only dependent on obstacle geometry, while laminar systems showed greater dependence on incoming flow conditions. The direct numerical simulations (DNS) of Vinuesa et al. (2015) compared laminar Blasius incoming boundary layer conditions to TBL conditions for a square cylinder. In their study, the boundary layer did not exceed $0.25 h$ and thus the top of the cylinder was in the approximately laminar free-stream for both cases. While they did find that the horseshoe vortex centre location moved and some of the wake characteristics varied as a result of the differing conditions, there was no change in the characteristic shedding frequency of the body (near $S t=0.1$ ). Hussein \& Martinuzzi (1996) and Meinders et al. (1999) both investigated cubes in turbulent channel flow. The former focussed on the dissipation and energy budget in various regions surrounding the cube, and the latter investigated heat transfer from the cube to the surrounding flow and its dependence on the dynamics associated with the geometry. However, no previous study has investigated the effects of turbulence intensity (separate from shear) on the wake characteristics of a cube. This is likely because it is non-trivial to generate a TBL where the turbulence parameters and mean velocity profile can be varied independently.

One way to address this issue is by introducing free-stream turbulence (FST) above the TBL (Charnay et al. 1976; Castro 1984; Hancock \& Bradshaw 1989; Sharp et al. 2009; Dogan et al. 2016). Two of the main effects of FST on the bulk characteristics of a TBL are that increasing $u_{\infty}^{\prime} / U_{\infty}$ increases the local turbulence intensity within the boundary layer, and it creates a fuller boundary layer profile, i.e., higher mean streamwise velocities closer to the wall. Interestingly, it appears that the mean velocity boundary layer profile 
eventually becomes saturated with increasing $u_{\infty}^{\prime} / U_{\infty}$; that is, increasing $u_{\infty}^{\prime} / U_{\infty}$ beyond a certain threshold no longer changes the mean velocity profile. However, in such cases, the turbulence intensity profile is still dependent on the FST (inferred from the results of Sharp et al. (2009) and Dogan et al. (2016)). This effect can be exploited to address varying $u_{h}^{\prime} / U_{h}$ while keeping the normalised shear approximately constant. The nondimensional shear investigated here is that normalised by the time-scale of the flow at the cube height $\left(h / U_{h}\right)$, which results in $\hat{\tau}_{h}=\left.\frac{h}{U_{h}} \frac{\partial U}{\partial y}\right|_{h}$. This quantity is particularly relevant when considering the case of buildings in the atmospheric boundary layer where measurements at the building height are attainable, while free-stream ones may not be. Furthermore, the parameter space made-up of $u_{h}^{\prime} / U_{h}$ and $\hat{\tau}_{h}$ contains information on the major aspects of the flow at the cube height, including: the turbulence intensity, the mean velocity, the shear, and the characteristic dimension.

This work identifies the effects of $u_{h}^{\prime} / U_{h}$ independently of $\hat{\tau}_{h}$ by fixing parameters which are known to affect the wake characteristics of a cube and the boundary layer development. First, $h / \delta \approx 0.5$ was fixed in order to disassociate any phenomenological differences from case-to-case from the relative position in the boundary layer. Second, $U_{\infty}$ was fixed and the cube was placed at the same streamwise position in order to maintain the same $R e_{x}=U_{\infty} x / \nu$ and boundary layer development for all cases. Measurements were performed independently with both planar particle image velocimetry (PIV) in the streamwise/wall-normal plane and hot-wire anemometry in the wake in order to investigate the spatial characteristics of the flow and the frequency information related to any shedding phenomena.

\section{Experimental procedure}

Measurements were performed in the University of Southampton's suction wind tunnel. The working section of this tunnel is $0.9 \mathrm{~m} \times 0.6 \mathrm{~m} \times 4.5 \mathrm{~m}$. Free-stream turbulence was generated by a $11 \times 7$ active grid placed at the inlet of the working section. The use of an active grid was inspired by Sharp et al. (2009) who used a similar set-up. The active grid is composed of $55.9 \mathrm{~mm} \times 55.9 \mathrm{~mm}$ square wings mounted to round rods that are actuated by stepper motors in the style of Makita (1991). The square wings have round holes that remove $25 \%$ of the square's surface area (figure 1(a)); see Dogan et al. (2016) for more details on the active grid. The boundary layer was formed on an artificial floor beginning $0.29 \mathrm{~m}$ downstream of the grid. A trailing edge flap and the height of the artificial floor were used to position the stagnation point on the tip of the leading edge of the artificial floor and to create an approximately zero-pressure-gradient. A 'zig-zag' roughness strip, located just downstream of the leading edge, was used to trip the boundary layer. For each test, the centre of the cube was located $2.7 \mathrm{~m}$ downstream from the leading edge of the artificial floor. A schematic of the set-up is provided in figure 1(b).

Four test cases with increasing free-stream turbulence intensity $\left(u_{\infty}^{\prime} / U_{\infty}\right)$ were investigated for constant $h / \delta \approx 0.47$ and $R e_{x} \approx 1.83 \times 10^{6}\left(U_{\infty} \approx 10 \mathrm{~m} / \mathrm{s}\right)$. One of the primary effects of FST on a TBL for constant $R e_{x}$ is that $\delta$ grows (table 1 ). As such, the size of the cube was varied with $u_{\infty}^{\prime} / U_{\infty}$ in order to keep $h / \delta$ approximately constant. The size of each cube is provided in table 1 along with $\delta$, which was estimated as $\delta_{0.99}$ from the region at least $3 h$ upstream of the cube. The values of $h / \delta$ and $R e_{x}$ for each case are also provided in table 1 along with the Reynolds numbers based on the velocity at the cube height, $R e_{h}=U_{h} h / \nu$, and the momentum thickness, $R e_{\theta}=U_{\infty} \theta / \nu$, for comparison to other studies. Castro \& Robins (1977) found that there were no discernible Reynolds number 


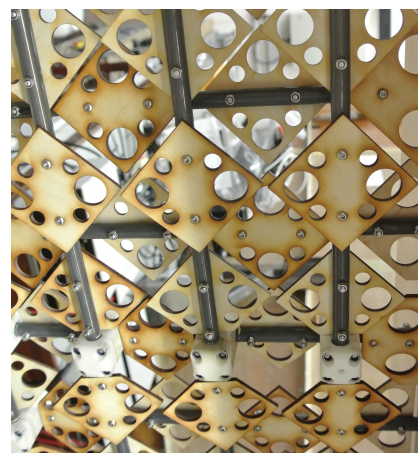

(a)

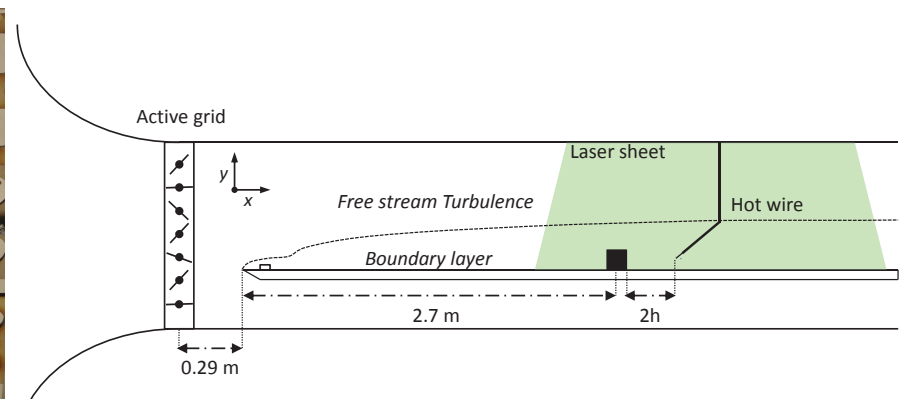

(b)

FiguRE 1. Experimental design: (a) active grid wings, and (b) schematic of measurement apparatus.

\begin{tabular}{ccccccccccccc} 
& $\begin{array}{c}u_{\infty}^{\prime} / U_{\infty} \\
{[\%]}\end{array}$ & $R e_{\lambda, \infty}$ & $\begin{array}{c}h \\
{[\mathrm{~mm}]}\end{array}$ & $\delta$ & $h / \delta$ & $\begin{array}{c}R e_{x} \\
{\left[\times 10^{6}\right]}\end{array}$ & $\begin{array}{c}R e_{\theta} \\
{\left[\times 10^{3}\right]}\end{array}$ & $\begin{array}{c}R e_{h} \\
{\left[\times 10^{3}\right]}\end{array}$ & $\begin{array}{c}U_{h} / U_{\infty} \\
{[\mathrm{mm}]}\end{array}$ & $\begin{array}{c}u_{h}^{\prime} / U_{h} \\
{[\%]}\end{array}$ & $\hat{\tau}_{h}$ & $L_{h} / h$ \\
\hline $\mathrm{A}$ & 0.5 & - & 19.7 & 41 & 0.48 & 1.86 & 3.2 & 11.4 & 0.85 & 5.4 & 0.20 & 0.40 \\
$\mathrm{~B}$ & 3.7 & 45 & 25.0 & 53 & 0.47 & 1.86 & 3.2 & 15.9 & 0.92 & 6.3 & 0.11 & 0.34 \\
$\mathrm{C}$ & 7.1 & 223 & 43.0 & 94 & 0.46 & 1.81 & 4.6 & 26.9 & 0.94 & 7.3 & 0.10 & 0.19 \\
$\mathrm{D}$ & 8.9 & 413 & 45.6 & 100 & 0.46 & 1.82 & 4.8 & 28.8 & 0.94 & 9.0 & 0.09 & 0.18
\end{tabular}

TABLE 1. Free-stream and incoming flow conditions for all test cases. Free-stream turbulence statistics were measured by the hot-wire probe at $y=212 \mathrm{~mm}$, which is $>2 \delta$ for all cases. The boundary layer thickness, $\delta=\delta_{0.99}$, and the turbulent statistics at the cube height were measured with PIV. The incoming $\delta$ and $L_{u}$ were assessed at least $3 h$ upstream of the cube.

effects for turbulent incoming conditions if $R e_{h}>4,000$ and the present measurements exceed this $R e_{h}$ by an order of magnitude. Additionally, we have used a trip that is most like that which produced the 'standard' canonical results in Marusic et al. (2015), and $R e_{\theta}>2,000$ for all cases which is within the range where the influence of the tripping conditions are negligible according to Schlatter \& Örlü (2012).

The four tests of table 1 have increasing free-stream turbulence intensity: $u_{\infty}^{\prime} / U_{\infty}=$ $0.5 \%, 3.7 \%, 7.1 \%$ and $8.9 \%$. The lowest turbulence intensity case, A, represents a canonical TBL with approximately no FST. This flow was generated without the active grid and is typically excluded from comparison to the other cases because its shear and velocity profile differ significantly from the others. However, it is presented as a canonical baseline. The $u_{\infty}^{\prime} / U_{\infty}=3.7 \%$ case, $\mathrm{B}$, was generated with the active grid in place, but held in a static position with half the wings perpendicular to the mean flow direction and the other half parallel to the mean flow direction, in an alternating pattern. The two higher turbulence intensity cases, $\mathrm{C}$ and $\mathrm{D}$, were generated with the active grid operating in the 'fully random' mode described by Hearst \& Lavoie (2015) with each motor receiving its own random top-hat distribution of time between updating motor commands $(0.05 \mathrm{~s} \leqslant t \leqslant 2.5 \mathrm{~s})$, wing acceleration $(50 \mathrm{~Hz} / \mathrm{s} \leqslant \alpha \leqslant 200 \mathrm{~Hz} / \mathrm{s})$, and wing velocity $(5 \mathrm{~Hz} \leqslant \omega \leqslant 15 \mathrm{~Hz}$ for case $\mathrm{C}$, and $1 \mathrm{~Hz} \leqslant \omega \leqslant 3 \mathrm{~Hz}$ for case $\mathrm{D})$.

For the PIV measurements, the flow was seeded with smoke droplets (diameter $\sim 1 \mu \mathrm{m}$ ). Two pulsed Litron Nd-YAG lasers (532 nm, $200 \mathrm{~mJ}$ ) were used simultaneously to create 
an extended laser sheet in the streamwise/wall-normal plane along the cube's axis of symmetry (centreline). Three adjacent LaVision ImagerProLX 16 mega-pixel cameras equipped with Sigma DG $105 \mathrm{~mm}$ lenses were oriented along the streamwise direction to capture the upstream flow and the wake of the cube. The resulting field-of-view (FOV) was $200 \mathrm{~mm}$ high, and $700 \mathrm{~mm}$ long. Vector fields were computed with DaVis 8.2 .2 on a GPU using multiple passes beginning with a coarse grid (64 pixels $\times 64$ pixels) and refined to a fine grid ( 24 pixels $\times 24$ pixels). Using an overlap of $50 \%$, the distance between two adjacent vectors is approximately $0.7 \mathrm{~mm}$. The characteristic dimension of the cube, $h$, is described by a minimum of 28 vectors for the worst case. Two thousand image pairs were acquired at an acquisition frequency of $0.25 \mathrm{~Hz}$, which resulted in all vector fields representing independent samples.

Hot-wire anemometry measurements were performed with an automated traverse and a Dantec StreamLine constant temperature anemometer. A single-wire probe $(5 \mu \mathrm{m}$ diameter tungsten wire; $1 \mathrm{~mm}$ sensing length), made in-house and mounted to Dantecstyle prongs, was used to perform the measurements. The wire was calibrated in situ using a 12 point variable exponent King's Law. Data were acquired for 7 minutes at a sampling frequency of $f_{s}=70.2 \mathrm{kHz}$ with an analog filter set at $f_{c}=30.0 \mathrm{kHz}$. This resulted in a minimum of 40,000 boundary layer turn-overs, which is ample data to converge the spectra and second-order statistics in both the free-stream and the boundary layer. Hotwire measurements were performed at five wall-normal locations $2 h$ downstream of the cube: $y / h=0.75,1.0,1.5,2.0$, and at a fixed free-stream position $(y=212 \mathrm{~mm})$, which is approximately equidistant from the top wall and the artificial floor, and greater than $2 \delta$ in all cases. The post-processing used here is described in detailed by Hearst \& Lavoie (2014). Gradient quantities were estimated from the hot-wire data using a sixth-order centred-difference scheme, which offers a good balance between accuracy and high-frequency filtering for hot-wire measurements (Hearst et al. 2012).

\section{Incoming flow conditions}

The incoming mean velocity $\left(U_{i}\right)$, turbulence intensity $\left(u_{i}^{\prime}\right)$, and Reynolds shear stress $\left(u^{\prime} v_{i}^{\prime}\right)$ profiles measured with PIV are given for each case in figures 2(a), (b), and (c), respectively (subscript $\cdot_{i}$ denotes incoming conditions). As $u_{\infty}^{\prime} / U_{\infty}$ was increased, $\delta$ increased (table 1) and the boundary layer became fuller. In addition, as the FST was increased, the turbulence intensity profile had less variation and a higher turbulence intensity was achieved closer to the wall (figure 2(b)). Figure 2(c) shows that the greatest magnitude Reynolds shear stress at the cube height occurs for the canonical case, and that it monotonically decreases with increasing $u_{\infty}^{\prime} / U_{\infty}$. The changes in $u^{\prime} v_{i}^{\prime}$ between cases are marginal and the effect of them on the wakes is investigated in the subsequent section. The difference in the profile for case B is likely related to the high static blockage of the unactuated grid. However, as will be shown, $u^{\prime} v_{i}^{\prime}$ does not have a significant impact on the wake flow. For the three test cases with added FST (B, C, D), the relative velocity and mean shear at the cube height changed marginally, $0.92 \leqslant U_{h} / U_{\infty} \leqslant 0.94$ and $0.09 \leqslant \hat{\tau}_{h} \leqslant 0.11$, respectively (table 1 ). Furthermore, the non-dimensional momentum in the mean profile beneath the cube height, $\int_{0}^{1} \frac{U}{U_{\infty}} \mathrm{d}\left(\frac{y}{h}\right)$, was between 7.6 and 7.9 for cases B, C, and D, compared to 6.7 for case A. The FST cases experienced an increase in local turbulence intensity of $6.3 \% \leqslant u_{h}^{\prime} / U_{h} \leqslant 9.0 \%$ with increasing $u_{\infty}^{\prime} / U_{\infty}$ (table 1 ). Thus, there are three cases $(\mathrm{B}, \mathrm{C}, \mathrm{D})$ with roughly $U_{h} / U_{\infty} \approx 0.93, \hat{\tau}_{h} \approx 0.1$ and a similar degree of incoming momentum, but a range of $u_{h}^{\prime} / U_{h}$, allowing us to comment on the effect of $u_{h}^{\prime} / U_{h}$ independently of mean velocity profile characteristics. In contrast, case A 


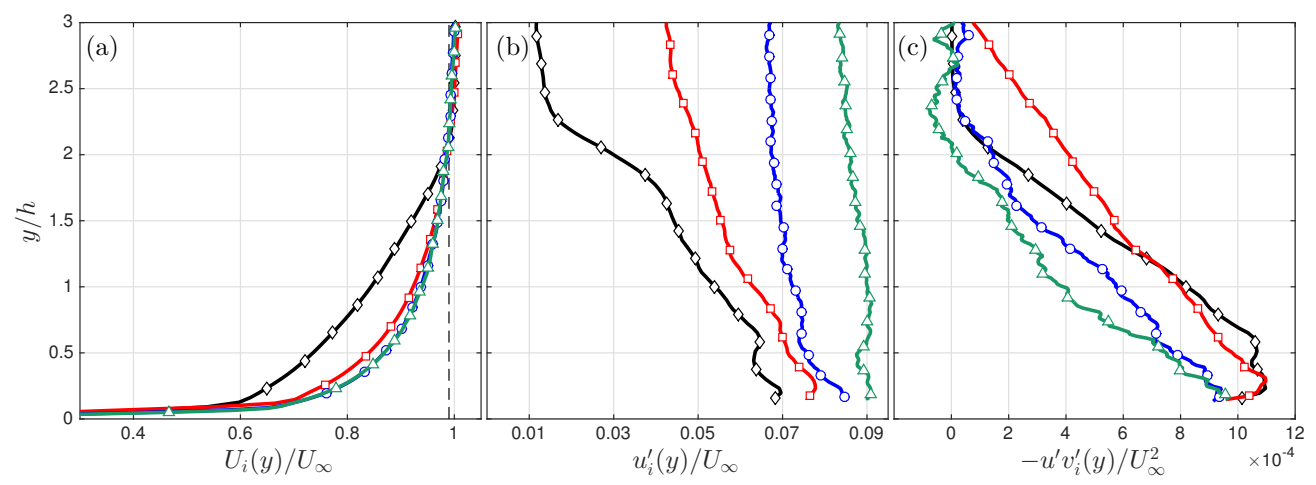

Figure 2. Profiles of the incoming turbulent flow: (a) incoming mean velocity, (b) incoming turbulence intensity, and (c) incoming Reynolds shear stress. Cases: $(\diamond)$ A, $(\square)$ B, $(\bigcirc)$ C, and $(\triangle) \mathrm{D}$.

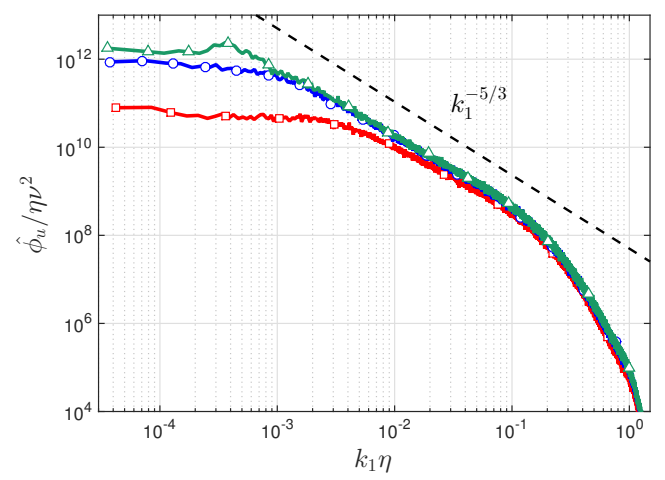

FiguRE 3. Kolmogorov variable normalised free-stream wavenumber spectra for turbulent cases: $(\square) \mathrm{B},(\bigcirc) \mathrm{C}$, and $(\triangle) \mathrm{D}$.

has a normalised shear level that is a factor of 2 different from the FST cases and as such it is not directly included in the turbulence intensity comparison.

Table 1 also includes estimates of the incoming integral length scale at the cube height $\left(L_{h}\right)$ estimated from PIV at least $3 h$ upstream of the cube. Here, $L$ is estimated by integrating the autocorrelation to the first zero-crossing. The zero-crossings of the correlations were similar to those reported by Ganapathisubramani et al. (2005). Despite the jump to higher $L_{h} / h$ for cases A and B relative to C and D, $L_{h} / h \leqslant 0.4$ for all cases, which is within the range that previous studies observed no significant effect of $L$ (Hillier \& Cherry 1981; Li et al. 2014).

Figure 3 shows the one-dimensional velocity spectra in wavenumber space, $\hat{\phi}_{u}\left(k_{1}\right)$, normalised by Kolmogorov variables for the three FST cases; $\eta=\nu^{3 / 4} /\langle\epsilon\rangle^{1 / 4}$ is the Kolmogorov microscale where $\langle\epsilon\rangle=15 \nu\left\langle(\partial u / \partial x)^{2}\right\rangle$ is the dissipation rate of turbulent kinetic energy assuming isotropy. For the hot-wire, Taylor's frozen flow hypothesis was employed, that is $(\partial / \partial t)=U(\partial / \partial x)$. The velocity wavenumber spectra show a near, but not exact, $k_{1}^{-5 / 3}$ range for the FST cases that spans a larger wavenumber space and better approximates $k_{1}^{-5 / 3}$ as $R e_{\lambda, \infty}=u_{\infty}^{\prime} \lambda_{\infty} / \nu$ is increased, where $\lambda^{2}=\left\langle u^{2}\right\rangle /\left\langle(\partial u / \partial x)^{2}\right\rangle$ is the square of the Taylor microscale. This result is in agreement with previous active grid studies (Mydlarski \& Warhaft 1996; Hearst \& Lavoie 2015), and validates that there is a fully developed turbulent free-stream above the TBL. 


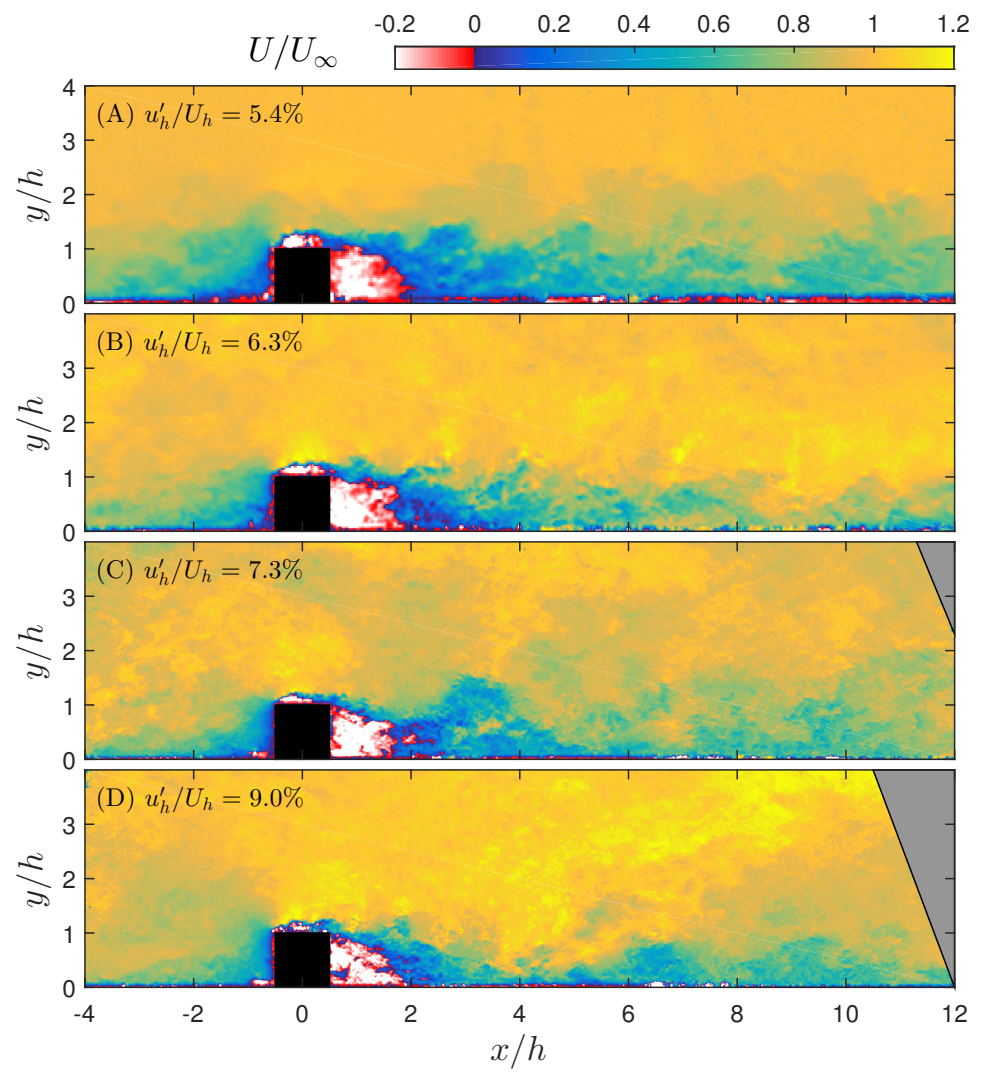

FiguRE 4. Sample instantaneous streamwise velocity fields for each test case.

\section{Spatial characteristics of the wake}

Representative instantaneous streamwise velocity fields from the PIV measurements are shown in figure 4. This figure provides the first insight into the flow phenomenology, and the differences and similarities between cases. A similarity between all three cases is that the flow appears to be attached to the top surface, at least near the trailing edge. Key indicators of this are the positive streamwise velocity after the negative separation bubble on the top surface of the cube, and the lack of a distinct shear layer leaving the downstream top edge of the cube. Also visible is the difference in the incoming flow field between the cases. In particular, the canonical case differs in that there is a larger momentum deficit upstream of the cube instantaneously than the more turbulent cases, which is supported by the profiles provided in figure 2. Lastly, in the wakes, the deficit region appears to be closer to the cube for increasing $u_{h}^{\prime} / U_{h}$. It thus seems relevant to both consider the mean velocity fields as well as compare the statistics of the flow fields with the incoming profile removed in order to ascertain how rapidly the flow returns to its upstream state. Furthermore, with respect to the attachment of the flow to the top surface, statistics are required to adequately interpret the flow state on the top surface of the cube.

The mean flow field obtained from the PIV measurements is shown for each case in figure 5, where mean streamlines are superimposed on the mean streamwise velocity fields. In general, the flow structure in the immediate proximity of the cube is not strongly dependent on the incoming flow conditions investigated here. From the mean 


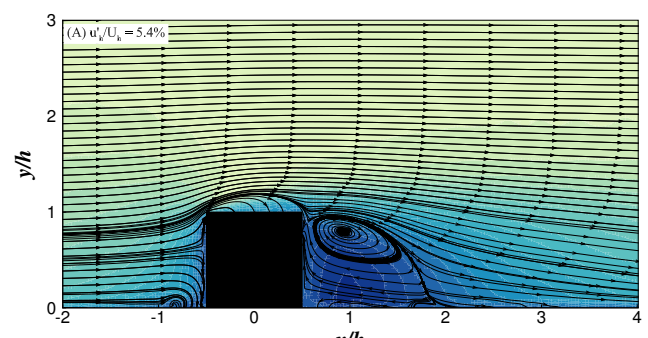

$x / h$

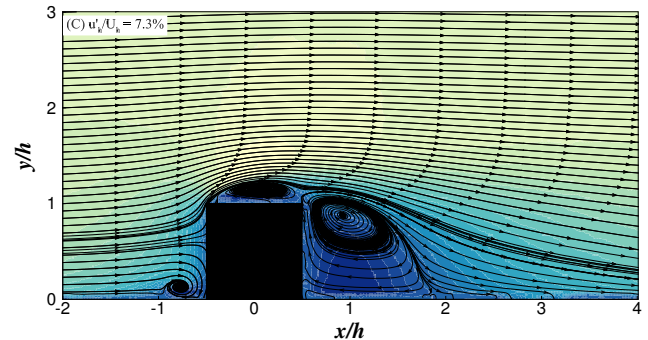

\section{$\boldsymbol{U} / \boldsymbol{U}_{\infty}$}

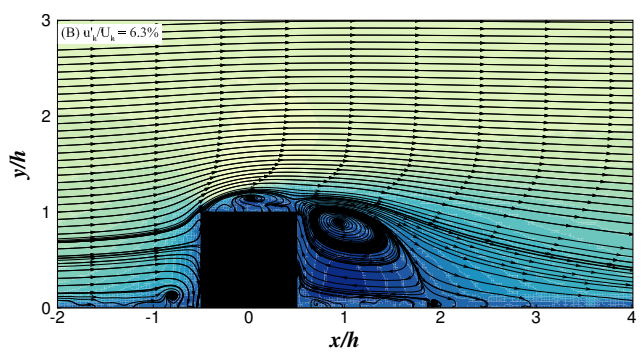

$x / h$

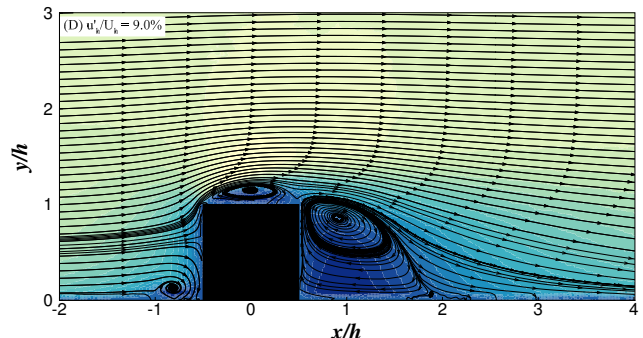

$\boldsymbol{x} / \boldsymbol{h}$

FiguRE 5. Mean streamwise velocity fields for each case with superimposed mean streamlines.

$\begin{array}{lllllll}u_{h}^{\prime} / U_{h} & \hat{\tau}_{h} & \overline{y_{0}} / h & \overline{x_{R}} / h & \tilde{x}_{t} / h & \gamma_{t} & S t\end{array}$

$[\%]$

$\begin{array}{llllllll}\mathrm{A} & 5.4 & 0.20 & 0.66 \pm 0.02 & 1.90 & 0.35 & 0.24 \pm 0.11 & 0.14 \\ \mathrm{~B} & 6.3 & 0.11 & 0.66 \pm 0.02 & 1.87 & 0.48 & 0.48 \pm 0.06 & 0.13 \\ \mathrm{C} & 7.3 & 0.10 & 0.63 \pm 0.01 & 1.90 & 0.46 & 0.45 \pm 0.04 & 0.13 \\ \mathrm{D} & 9.0 & 0.09 & 0.65 \pm 0.01 & 1.88 & 0.44 & 0.41 \pm 0.04 & -\end{array}$

TABLE 2. Characteristics of the flow in the immediate proximity of the wall-mounted cubes.

The uncertainties in position are the same for $\overline{y_{0}} / h, \overline{x_{R}} / h$ and $\tilde{x}_{t} / h$.

streamline figures, a stagnation point $\left(y_{0}\right)$ on the front face of the cube was observed at an approximate mean height $\overline{y_{0}} / h \approx 0.65$ for all cases (within uncertainty), and thus did not show a strong dependence on either $u_{h}^{\prime} / U_{h}$ or $\hat{\tau}_{h}$ (table 2). Downstream of the cube, the flow was characterised by a recirculation region. The mean reattachment point of this region was estimated from the streamline plot, and was $\overline{x_{R}} / h=1.9$ for all cases (table 2), which is also independent of $u_{h}^{\prime} / U_{h}$ and $\hat{\tau}_{h}$; this is $1.4 h$ downstream of the rear face of the cube. This location is consistent with that observed in the wake of a cube in channel flow (Hussein \& Martinuzzi 1996), and the relative independence of its position with changing turbulence intensity is supported by the measurements of Ballio et al. (1998).

Above the cube, a recirculation bubble forms due to the separating shear layer at the leading edge. The separated flow attaches to the top surface of the cube intermittently. The 'reattachment' of the shear layers formed on a cube was previously observed for cubes immersed in a turbulent flow (Castro \& Robins 1977; Meinders et al. 1999), although we use the terminology 'attach' to the top surface to distinguish this phenomenon from that in the wake and because the flow was not initially attached to the top surface. The 
intermittency of attachment to the top surface is one of the first signs of the effects of varying the incoming conditions. The intermittency for each case is provided in table 2 with uncertainty estimates that vary with the resolution $\left(\gamma_{t}=0\right.$ implies the flow always attaches to the top surface). For cases B, C, and D at the same $\hat{\tau}_{h} \approx 0.1$, the flow more frequently attaches to the top surface, and thus the intermittency decreases, with increasing $u_{h}^{\prime} / U_{h}$. This corresponds to the median point of attachment to the top surface $\left(\tilde{x}_{t} / h\right)$, which approaches the leading edge of the cube as the intermittency drops and $u_{h}^{\prime} / U_{h}$ increases. This agrees with observations of the separation and attachment on a thick flat plate subjected to FST (Saathoff \& Melbourne 1997). We attribute the significant differences in intermittency between the canonical case A, and the FST cases to the difference in $\hat{\tau}_{h}$.

The same technique as Pearson et al. (2013) was employed to determine the reattachment location in the wake of the cube, as well as the attachment location and intermittency on the top surface. Our particular application of this technique was to look for the first position where instantaneously $U<0$ against the boundary while scanning from a downstream position towards an upstream position. This is similar to how mean recirculation was determined in previous studies with hot-wires or tufts (Antoniou \& Bergeles 1988). The intermittency was inferred from assessing the percentage of samples in which an attachment point was found on top of the cube.

Upstream of the cubes, a horseshoe vortex forms. The centre location of the horseshoe vortex also appears to show some dependence on the incoming conditions. For canonical case A, the horseshoe vortex is centred near $(x / h, y / h)=(-0.8,0.04)$, while for the three FST cases it is centred near $(x / h, y / h)=(-0.8,0.1)$. The latter is similar to the horseshoe vortex core location reported by Lim et al. (2009) for LES of a TBL immersed cube. Furthermore, for turbulent incoming conditions, Baker (1980) found that the centre of the horseshoe vortex occurred near $x / h=-0.8$ and that its position was independent of Reynolds number. The rising of the horseshoe vortex core away from the wall for increased turbulence was also observed by Vinuesa et al. (2015) in their DNS comparing incoming Blasius profile boundary layers to canonical TBLs interacting with the lower part of a square cylinder. However, we note that for our three cases with approximately constant $\hat{\tau}_{h}$, but increasing $u_{h}^{\prime} / U_{h}$, the core does not appreciable change location, suggesting its movement is linked to mean shear rather than turbulence intensity.

Figure 6 shows the streamwise velocity field with the incoming profile (figure 2) removed for all cases. Contours at $\left|\left(U-U_{i}\right) / U_{\infty}\right|=0.05$ are included in figure 6 . This threshold was chosen as a relative indicator of mean wake length, and in general the conclusions are not sensitive to the choice of threshold. It was also chosen to be at approximately $u_{h}^{\prime} / U_{h}$ of the lowest turbulence intensity case (case A). First, consider the three cases at the same normalised shear $\hat{\tau}_{h} \approx 0.1(\mathrm{~B}, \mathrm{C}, \mathrm{D})$. The two higher $u_{h}^{\prime} / U_{h}$ cases $(\mathrm{C}$ and $\mathrm{D})$ both have a smaller deficit region upstream of them relative to case B. Downstream of the cube, the longest wake (with respect to the mean streamwise velocity deficit) occurs for case B. For B, the -0.05 contour level extends to a maximum streamwise location of $x / h=11.8$ compared to 8.6 for $\mathrm{C}$ and 8.2 for D. This illustrates a monotonic reduction in the wake length relative to case $\mathrm{B}$ of $27 \%$ and $31 \%$ for cases $\mathrm{C}$ and $\mathrm{D}$, respectively, at fixed $\hat{\tau}_{h} \approx 0.1$. These are substantial changes given the turbulence was only increased by $1 \%$ between case B and C, and $2.7 \%$ between $\mathrm{B}$ and $\mathrm{D}$. This wake reduction also corresponds to a reduction in $\gamma_{t}$ with $u_{h}^{\prime} / U_{h}$.

The difference between cases $\mathrm{B}$ and $\mathrm{C}$ is markedly more substantial than that between cases $\mathrm{C}$ and $\mathrm{D}$. This may suggest that the ability of the turbulent fluctuations to facilitate wake recovery saturates beyond a certain turbulence intensity. There are at least two possible causes of this. First, it is possible that there is a balance between the turbulence 

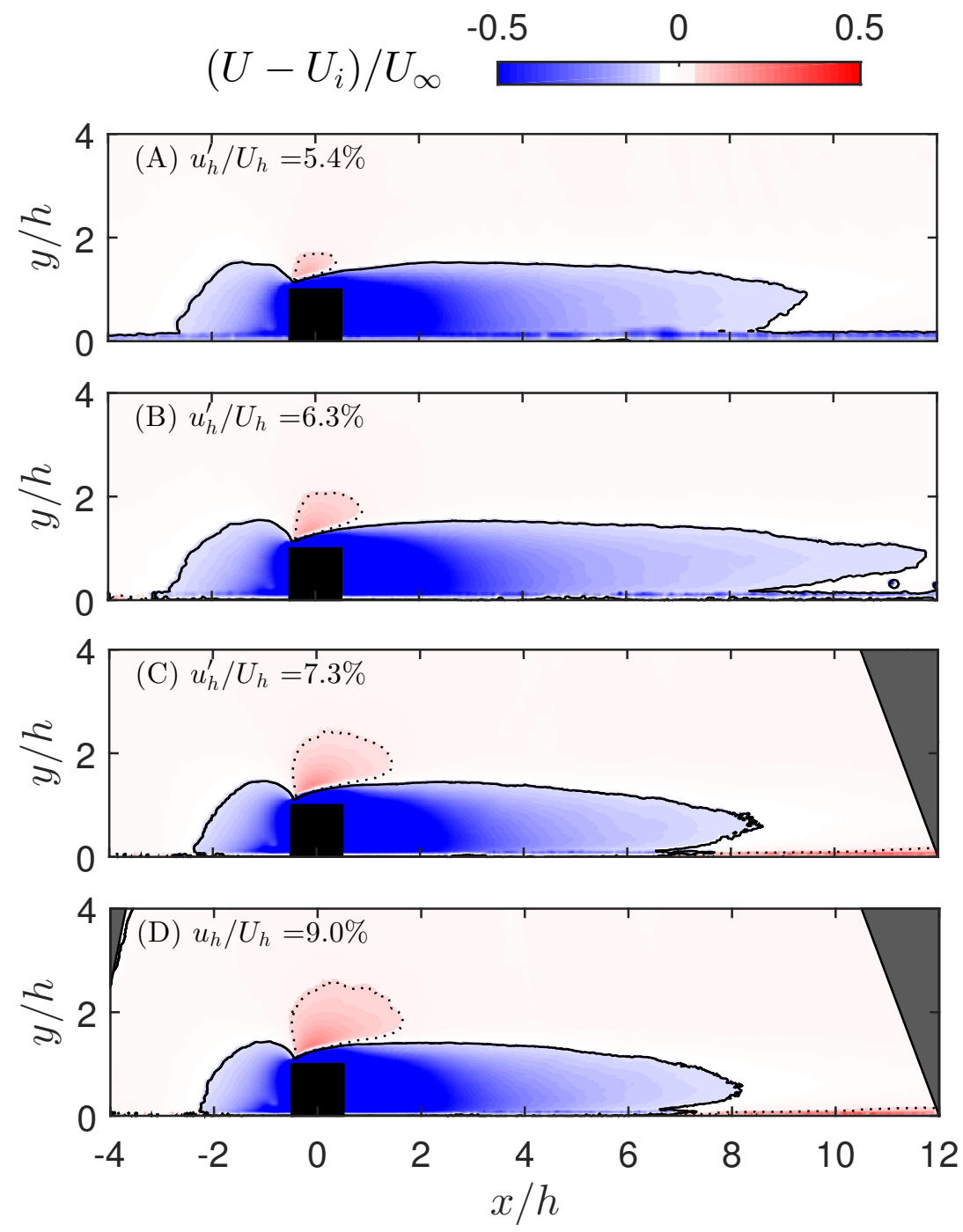

Figure 6. Mean streamwise velocity field with the incoming profile removed. Contour lines at: (dashed) +0.05 and (solid) -0.05 .

level at the height of the obstacle and the turbulence generated by the obstacle in its wake. Therefore, beyond a certain level it might not be possible to alter the entrainment in the wake any further, resulting in a system of diminishing returns as $u_{h}^{\prime} / U_{h}$ is increased. Alternatively, the significant difference may be related to the size of the incoming large scales at the cube height, which is $L_{h} / h=0.34$ for case $\mathrm{B}$, but $L_{h} / h \approx 0.19$ for cases $\mathrm{C}$ and D. While these are all below the threshold for which no significant effects were noted for the separation bubbles and drag forces in previous studies (Saathoff \& Melbourne 1997; Li et al. 2014), it is possible that the 1.5 times larger large scales for case B relative to cases $\mathrm{C}$ and $\mathrm{D}$ results in less frequent transport of high-speed fluid into the wake 
relative to $h$. Regardless, of the above, the results are significant in that they show a monotonic decrease in the mean wake deficit region with increasing $u_{h}^{\prime} / U_{h}$.

In figure 6 , the canonical case $\mathrm{A}$ has a shorter wake than $\mathrm{B}$. This is attributable to the significant difference between $U_{h} / U_{\infty}$ and $\hat{\tau}_{h}$ for A compared to B. In particular, case B has a much fuller profile at the cube height, with $U_{h} / U_{\infty}=0.92$, compared to $U_{h} / U_{\infty}=0.85$. For this range, the turbulence intensity varies by less than $1 \%$ between them. Thus, the distance required to recover the upstream velocity for case B is longer and the evidence suggests the turbulence intensity is of insufficient magnitude to facilitate wake recovery. In addition, the magnitude of $\hat{\tau}_{h}$ for case A is nearly twice that of B, because of the shallower slope of the velocity profile at the cube height (figure 2). This also indicates that there is lower incoming momentum for case A over the characteristic dimension $h$, and thus, for a similar amount of turbulent kinetic energy, the wake recovers to the same low flow state over less distance.

Figure 7 shows the normalised streamwise velocity fluctuation deficit relative to the incoming turbulence intensity profile. These maps allow for visualisation of the ratio of increase or decrease in the fluctuations compared to the incoming flow at each wall-normal position. Figure 7 highlights that the fluctuations recover to their original state closer to the cube for increasing $u_{h}^{\prime} / U_{h}$, in agreement with the results for the mean velocity. These results are suggestive that the increase in turbulence intensity at the cube height facilitates the transport of high momentum fluid into the wake, thus decreasing the wake length. This is corroborated in figure 5 by the strong downward velocity after the recirculation region in the wake of the cube that deflects the streamlines downward and that likely acts to entrain faster moving fluid into the wake from above. The increased turbulence intensity below $h$ for increasing $u_{h}^{\prime} / U_{h}$ suggests a similar mechanism would move high momentum fluid laterally into the wake as well.

Maps of the mean Reynolds shear stress are provided in figure 8. The disturbance to the incoming $u^{\prime} v^{\prime}$ profile extends beyond the measurement range of the present setup although $u^{\prime} v^{\prime}$ is clearly recovering at the end of the FOV. The wakes of the three cases at $\hat{\tau}_{h} \approx 0.1$ do not substantially differ with respect to $u^{\prime} v^{\prime}$, while canonical case A with $\hat{\tau}_{h}=0.2$ has a smaller $u^{\prime} v^{\prime}$ deficit region in its wake. This suggests that the $u^{\prime} v^{\prime}$ recovery is primarily dependent on mean shear rather than turbulence intensity. Despite the marginally differing incoming $u^{\prime} v^{\prime}$ profiles highlighted in figure $2(\mathrm{c})$, the wake appears to scale with this difference if $\hat{\tau}_{h}$ is maintained thus marginalising the importance of the incoming $u^{\prime} v^{\prime}$ profile for such cases.

\section{Time-series in the wake}

In the context of bluff bodies immersed in approximately homogeneous FST, increased turbulence intensity appeared to correlate with a reduction in the strength of vortex shedding motions (Blackburn \& Melbourne 1996; Rind \& Castro 2012). A similar phenomenon is investigated here with hot-wire anemometry measurements in the wake. The frequency domain velocity spectra, $\phi_{u}(f)$, normalised by large scale variables, are shown in figure $9(\mathrm{a})$ at $(x / h, y / h)=(2.5,0.75)$ for all cases; this wall-normal position was chosen to ensure the probe was completely immersed in the wake. Given that $\overline{x_{R}} / h$ is approximately fixed for all cases, the probe is in the same position relative to the primary wake circulation region. Note that the origin of the $x$-axis is at the centre of the cube, so this position is $2 h$ downstream of the rear face of the cube. In figure $9(\mathrm{a})$, a dominant non-dimensional shedding frequency is apparent for the lower turbulence intensity cases, $\mathrm{A}$ and $\mathrm{B}$, at a local Strouhal number of $S t=f h / U \approx 0.13$. This is similar to the dominant shedding peak at $S t \approx 0.1$ observed in experiments (Wang \& Zhou 2009; Sattari et al. 

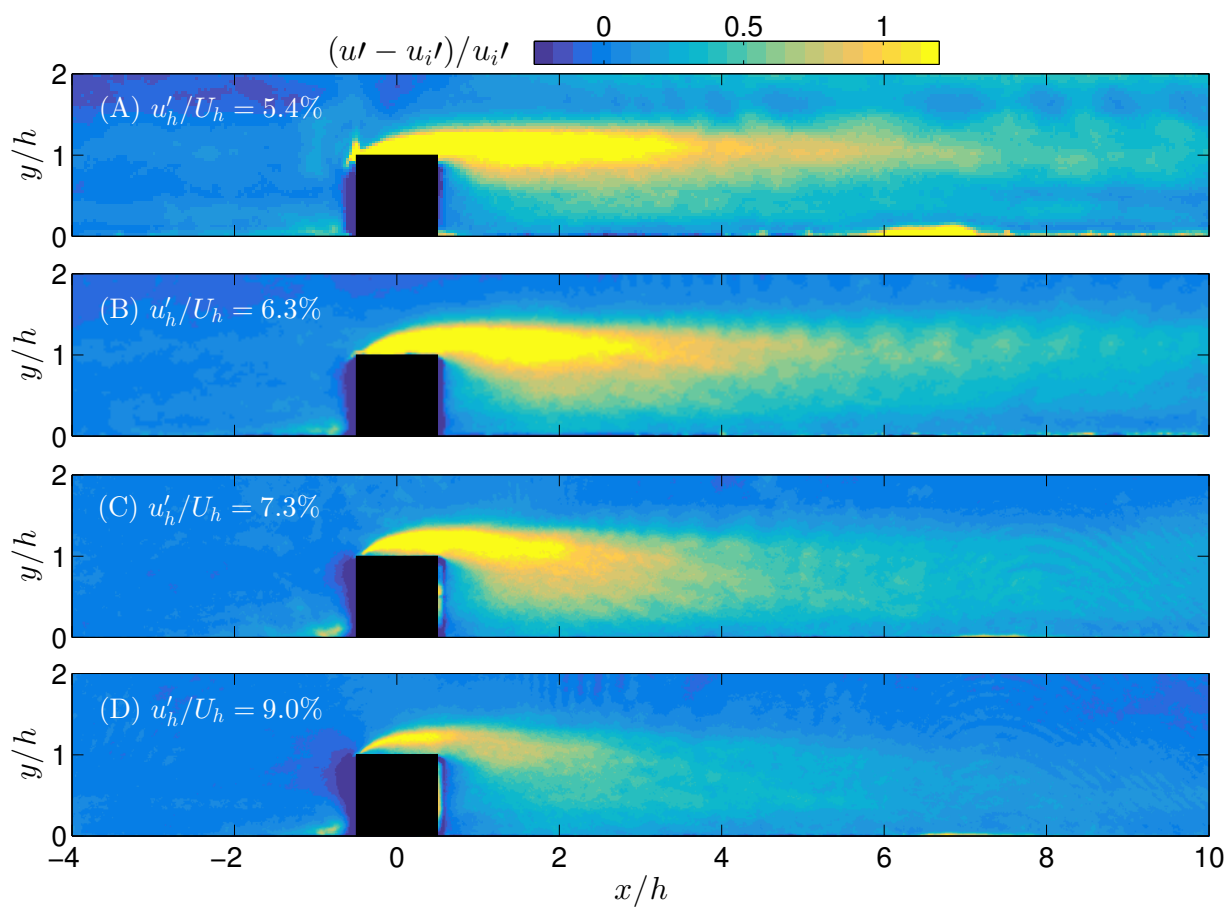

FigURE 7. Streamwise velocity fluctuations compared to the incoming velocity fluctuation profile.

2012) and DNS (Vinuesa et al. 2015) of a variety of square cylinders, and $S t=0.145$ observed by Hussein \& Martinuzzi (1996) for a cube in turbulent channel flow. This peak must be related to flow induced by the presence of the cube because there is no peak near $S t=0.13$ in the incoming flow.

The shedding peak observed here has diminishing prominence and is less broadband with increasing $u_{h}^{\prime} / U_{h}$. Here, we use the word prominence to describe the magnitude of the shedding peak relative to (or above) the energy content of the other frequencies in the normalised spectra (figure $9(\mathrm{a})$ ). The unnormalised wake spectra are shown in figure 9 (b). From this figure it is apparent that the increased turbulence intensity results in higher energy levels at all scales $f h / U<1$, including those surrounding the shedding frequency, thus reducing the prominence of the shedding peak relative to the background flow. This also correlates to a reduction in the intermittency of the recirculation bubble on top of the cube for the FST cases (table 2). Regardless of whether the shedding is masked or eliminated by increasing $u_{h}^{\prime} / U_{h}$, the results suggest a suppression of the shedding motions at the $S t$ prevalent in the low turbulence cases. The existence of the $S t=0.13$ peak for case B and its diminishing presence for the higher cases suggests the shortening of the wake region for cases $\mathrm{C}$ and $\mathrm{D}$ is related to a reduction in the strength of the dominant shedding motions.

The pre-multiplied frequency spectra, $\phi_{u}(f)$, from the $y / h$ scan are shown in figure 10 for each test case. The pre-multiplied spectrum identifies the frequencies that carry the majority of the energy in the flow. Dogan et al. (2016) demonstrated that the FST imposes its own shape for $\phi_{u}$ on the outer regions of the boundary layer, and this spectral shape persists until nearly $y^{+}=100$, which is of order $\sim 0.1 \delta$. This is well below $h$ in the present experiment. Thus any change to $\phi_{u}$ as one descends through the boundary layer and below $h$ must be a result of the presence of the cube. 


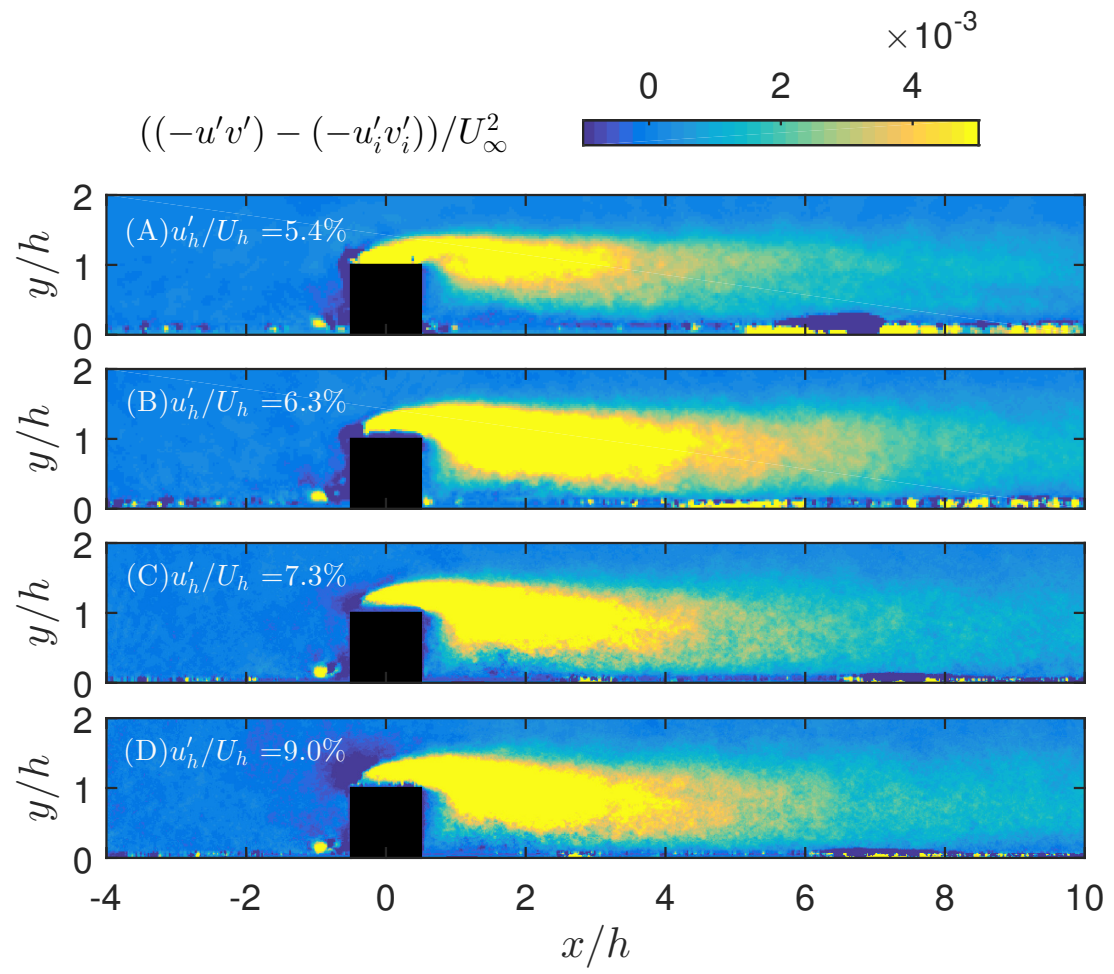

Figure 8. Mean Reynolds shear stress fields with the incoming mean Reynolds shear stress profile removed.

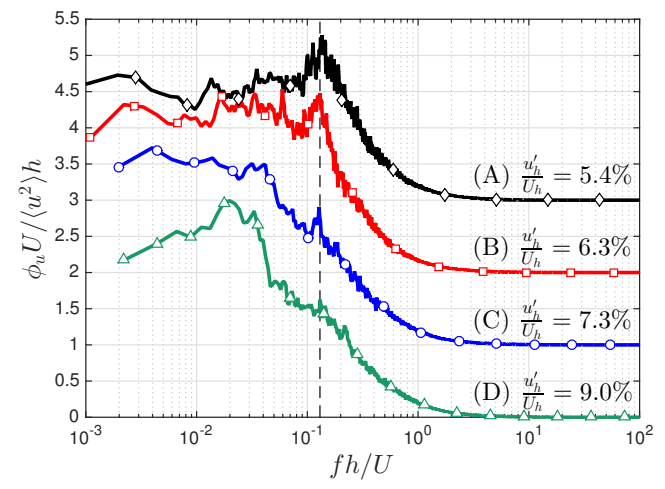

(a)

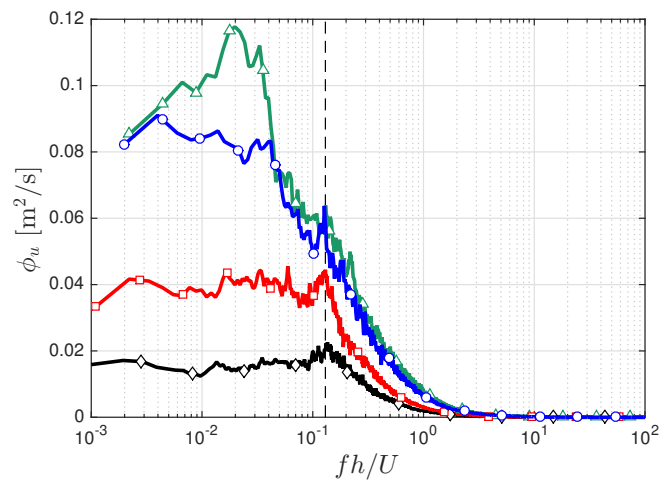

(b)

Figure 9. Frequency space velocity spectra at $(x / h, y / h)=(2.5,0.75)$ for all cases: (a) normalised by large scale variables, and (b) no normalisation. Dashed line is at $S t=0.13$. Cases $\mathrm{A}, \mathrm{B}$, and $\mathrm{C}$ are offset by $+3,+2$, and +1 , respectively, in (a).

Case D in figure 10 is a strong illustrative example of the physics at play as one enters the cube wake. The frequencies that carry the majority of the energy outside of the boundary layer are set by the FST and are identified by a peak near $f h / U=10^{-2}$. This peak is present at $y=2 h=0.92 \delta$ and $4.4 h=2 \delta$, and in the flows of Sharp et al. (2009) 

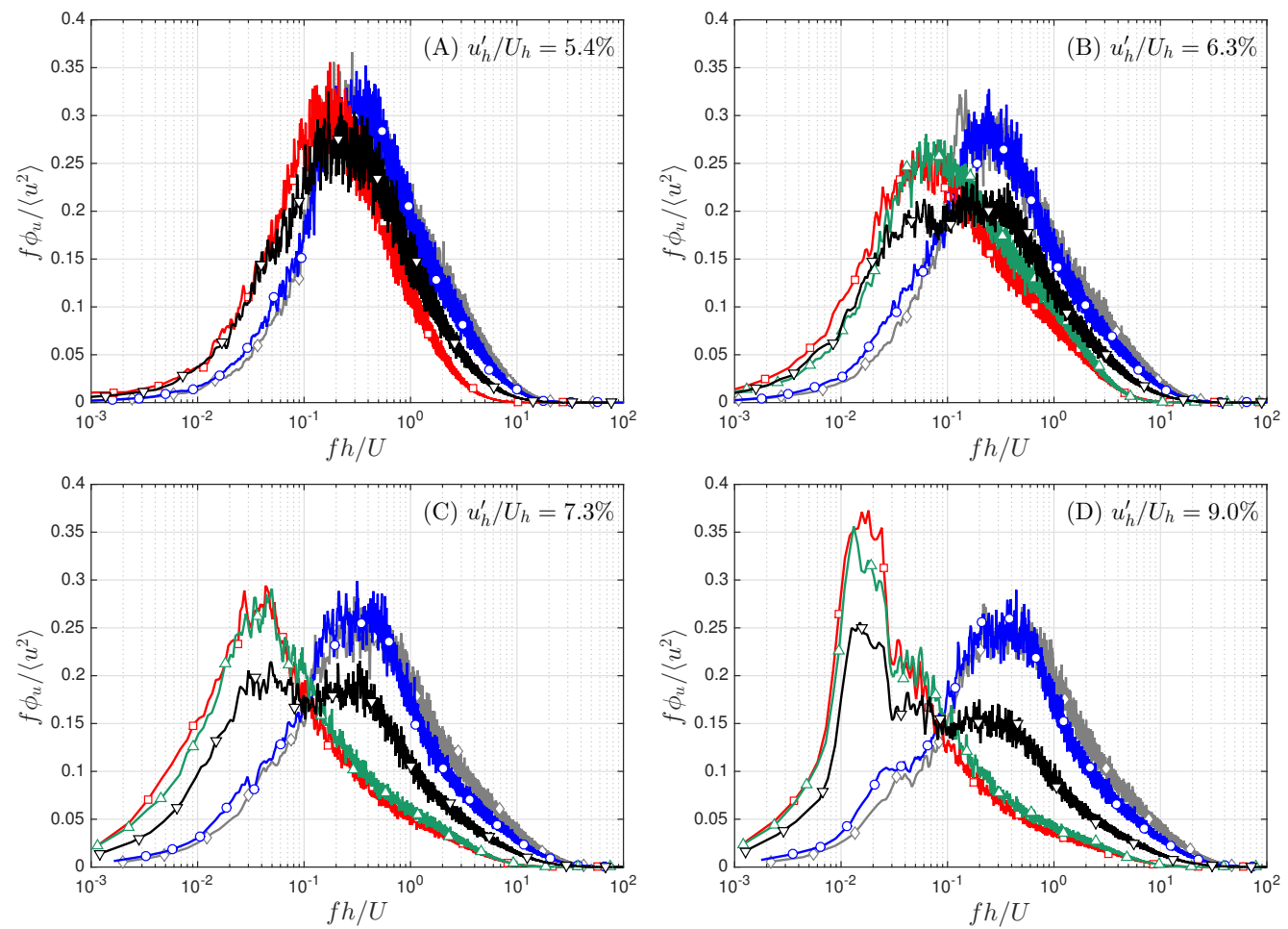

FiguRE 10. Pre-multiplied frequency spectra $2 h$ downstream of the cube for all cases; $(\diamond) y / h=0.75,(\bigcirc) y / h=1.0,(\nabla) y / h=1.5,(\square) y / h=2.0,(\triangle)$ free-stream station.

and Dogan et al. (2016) persisted all the way to the near-wall region. However, in the present experiment, this energy is redistributed to frequencies near $f h / U=S t=0.13$ as one descends into the cube wake $(y / h=0.75$ and 1.0). At $y / h=1.5$, a clear transition between the two regions is apparent and the spectrum exhibits both the outer peak and the wake peak. The other three cases follow the same trend as D. That is, the FST imposes a shape and an outer peak on the spectra, but as one moves down through the boundary layer into the wake of the cube, the spectrum of the wake dominates the flow, regardless of the outer layer spectrum. The spectra in the cube wake are remarkably similar despite having transitioned from entirely different incoming conditions. This supports the observation from the present and previous studies that the downstream reattachment point is not significantly influenced by the upstream flow if it is turbulent.

\section{Conclusions}

The effect of turbulence on the wake of a turbulent boundary layer immersed cube was investigated by creating turbulent boundary layers with similar shear profiles but different turbulence intensity profiles. This was done by subjecting the TBL to freestream turbulence, which allowed for a range of $6.3 \% \leqslant u_{h}^{\prime} / U_{h} \leqslant 9.0 \%$ to be investigated for relatively fixed $\hat{\tau}_{h} \approx 0.1$. These were also compared to a canonical case without FST where $u_{h}^{\prime} / U_{h}=5.4 \%$ and $\hat{\tau}_{h}=0.20$. Measurements were performed for a fixed $h / \delta=0.47$ and $R e_{x}=1.83 \times 10^{6}$.

The mean stagnation point on the upstream face and reattachment point in the wake of the cube were found to be independent of $u_{h}^{\prime} / U_{h}$ and constant at $\overline{y_{0}} / h=0.65$ and 
$\overline{x_{R}} / h=1.9$, respectively. A separated shear layer formed at the leading edge of the cube and attached to the top surface intermittently. For a fixed shear level of $\hat{\tau}_{h} \approx 0.1$, this intermittency decreased (i.e., the flow attached more frequently) with increasing $u_{h}^{\prime} / U_{h}$. This correlated to a monotonic reduction in wake length with increasing $u_{h}^{\prime} / U_{h}$ at constant $\hat{\tau}_{h} \approx 0.1$ as judged by the mean velocity and turbulent fluctuation deficits. When comparing wakes with different incoming shear levels, the wake length appeared to be dominated by the shear and the relative velocity at the cube height rather than the turbulence intensity, as the wake had more momentum to recover for a higher value of $U_{h} / U_{\infty}$ and a lower value of $\hat{\tau}_{h}$. Finally, when $\hat{\tau}_{h}$ was held constant the peak in the spectrum in the wake monotonically diminished, which identifies a weakening in the strength of the vortex shedding motions.

Future studies should focus on the forces and pressure field around the cube and compare these both to prior results for cubes in canonical turbulent boundary layers, e.g., Castro \& Robins (1977), and to the effects experienced by bluff bodies subjected directly to free-stream turbulence. Furthermore, the 3-dimensional effects of the varying turbulence levels can be more fully investigated by either using a 3D measurement technique (e.g., tomographic PIV) or performing further planar measurements in different streamwise and transverse planes. It would also be interesting to further explore the $\left(u_{h}^{\prime} / U_{h}, \hat{\tau}_{h}\right)$ parameter space, e.g., hold $u_{h}^{\prime} / U_{h}$ constant and vary $\hat{\tau}_{h}$. This parameterisation incorporates the major flow parameters around the cube and can be practically measured in situ in atmospheric applications, thus making it useful for comparisons to measurements in the atmospheric boundary layer.

The authors acknowledge the financial support of the European Research Council (ERC Grant agreement No. 277472), the Engineering and Physical Sciences Research Council of the United Kingdom (EPSRC Grant Ref No: EP/I037717/1), and the Leverhulme Trust. RJH acknowledges the financial support of the Natural Sciences and Engineering Research Council of Canada (NSERC). The authors are also grateful to Prof. I.P. Castro for his comments on the manuscript and numerous insightful discussions, and Mr. J. van der Kindere for auxiliary measurements. All data supporting the figures presented in this study are openly available from the University of Southampton repository at http://dx.doi.org/10.5258/SOTON/399645.

\section{REFERENCES}

Antoniou, J. \& Bergeles, G. 1988 Development of the reattached flow behind surfacemounted two-dimensional prisms. J. Fluids Eng. 110, 127-133.

BAKER, C. J. 1979 The laminar horseshoe vortex. J. Fluid Mech. 95 (2), 347-367.

BAKER, C. J. 1980 The turbulent horseshoe vortex. J. Wind Eng. Ind. Aero. 6, 9-23.

Ballio, F., Bettoni, C. \& Franzetti, S. 1998 A survey of time-averaged characteristics of laminar and turbulent horseshoe vortices. J. Fluids Eng. 120, 223-242.

Blackburn, H. M. \& Melbourne, W. H. 1996 The effect of free-stream turbulence on sectional lift forces on a circular cylinder. J. Fluid Mech. 306, 267-292.

CAStro, I.P. 1984 Effects of free stream turbulence on low Reynolds number boundary layers. J. Fluids Eng. 106 (3), 298-306.

Castro, I.P. \& Robins, A.G. 1977 The flow around a surface-mounted cube in uniform and turbulent streams. J. Fluid Mech. 79 (2), 307-335.

Charnay, G., Mathieu, J. \& Comte-Bellot, G. 1976 Response of a turbulent boundary layer to random fluctuations in the external stream. Phys. Fluids 19, 1261-1272.

Dogan, E., Hanson, R. \& Ganapathisubramani, B. 2016 Interactions of large-scale freestream turbulence with turbulent boundary layers. J. Fluid Mech. 802, 79-107.

Ganapathisubramani, B., Hutchins, N., Hambleton, W. T., Longmire, E. K. \& 
MARusic, I. 2005 Investigation of large-scale coherence in a turbulent boundary layer using two-point correlations. J. Fluid Mech. 524, 57-80.

HANCOCK, P. E. \& Bradshaw, P. 1989 Turbulence structure of a boundary layer beneath a turbulent free stream. J. Fluid Mech. 205, 45-76.

Hearst, R. J., Buxton, O. R. H., Ganapathisubramani, B. \& Lavoie, P. 2012 Experimental estimation of fluctuating velocity and scalar gradients in turbulence. Exp. Fluids 53 (4), 925-942.

Hearst, R. J. \& Lavoie, P. 2014 Decay of turbulence generated by a square-fractal-element grid. J. Fluid Mech. 741, 567-584.

Hearst, R. J. \& Lavoie, P. 2015 The effect of active grid initial conditions on high Reynolds number turbulence. Exp. Fluids 56 (10), 185.

Hillier, R. \& CherRy, N.J. 1981 The effect of stream turbulence on separation bubbles. J. Wind Eng. Ind. Aero. 8, 49-58.

Hussein, H. J. \& Martinuzzi, R. J. 1996 Energy balance for turbulent flow around a surface mounted cube placed in a channel. Phys. Fluids 8 (3), 764-780.

LI, Q.S., Hu, G. \& YAN, B. 2014 Investigation of the effects of free-stream turbulence on wind-induced responses of tall building by Large Eddy Simulation. Wind Struc. 18 (6), 599-618.

Lim, H.C., Castro, I.P. \& Hoxey, R.P. 2007 Bluff bodies in deep turbulent boundary layers: Reynolds-number issues. J. Fluid Mech. 571, 97-118.

Lim, H.C., Thomas, T.G. \& Castro, I.P. 2009 Flow around a cube in a turbulent boundary layer: LES and experiment. J. Wind Eng. Ind. Aero. 97, 96-109.

Makita, H. 1991 Realization of a large-scale turbulence field in a small wind tunnel. Fluid Dyn. Res. 8, 53-64.

Marusic, I., Chauhan, K. A., Kulandaivelu, V. \& Hutchins, N. 2015 Evolution of zeropressure-gradient boundary layers from different tripping conditions. J. Fluid Mech. 783, 379-411.

Meinders, E. R., Hanjalic, K. \& Martinuzzi, R. J. 1999 Experimental study of the local convection heat transfer from a wall-mounted cube in turbulent channel flow. J. Heat Transfer 121 (3), 564-573.

Mydlarski, L. \& WARHAFt, Z. 1996 On the onset of high-Reynolds-number grid-generated wind tunnel turbulence. J. Fluid Mech. 320, 331-368.

Pearson, D. S., Goulart, P. J. \& Ganapathisubramani, B. 2013 Turbulent separation upstream of a forward-facing step. J. Fluid Mech. 724, 284-304.

Rind, E. \& CASTRO, I.P. 2012 On the effects of free-stream turbulence on axisymmetric disc wakes. Exp. Fluids 53, 301-318.

SaAthoff, P.J. \& Melbourne, W.H. 1997 Effects of free-stream turbulence on surface pressure fluctuations in a separation bubble. J. Fluid Mech. 337, 1-24.

Sattari, P., Bourgeois, J. A. \& Martinuzzi, R. J. 2012 On the vortex dynamics in the wake of a finite surface-mounted square cylinder. Exp. Fluids 52, 1149-1167.

Schlatter, P. \& ÖRlü, R. 2012 Turbulent boundary layers at moderate Reynolds numbers: inflow length and tripping effects. J. Fluid Mech. 710, 5-34.

Sharp, N., Neuscamman, S. \& Warhaft, Z. 2009 Effects of large-scale free stream turbulence on a turbulent boundary layer. Phys. Fluids 21 (095105).

Vinuesa, R., Schlatter, P., Malm, J., Mavriplis, C. \& Henningson, D. S. 2015 Direct numerical simulation of the flow around a wall-mounted square cylinder under various inflow conditions. J. Turb. 16 (6), 555-587.

WANG, H. F. \& Zhou, Y. 2009 The finite-length square cylinder near wake. J. Fluid Mech. 638, 453-490. 